\title{
COMPARISON OF PERFORMANCES OF FUZZY LOGIC WITH ECHOSTATE NEURAL NETWORK FOR DIAGNOSIS AND PROGNOSIS OF SINGLE POINT TOOL WEAR ESTIMATION USING HYBRID ALUMINIUM SILICON CARBIDE METAL MATRIX COMPOSITE
}

\author{
${ }^{1}$ Kathirvel M., ${ }^{2}$ Palanikumar K. \\ ${ }^{1}$ Department of Mechanical Engg, Sun Engineering College, Nagarcoil, India \\ ${ }^{2}$ Principal, Sri Sairam Institute of Technology, Chennai, India
}

\begin{abstract}
In this research work, prognosis and diagnosis of tool wear for the polycrystal diamond (PCD) tool has been done by using fuzzy logic and Echostate Neural Network during machining of Al6061 metal matrix composite. Diagnosis refers to estimation of amount of tool worn out and prognosis refers to estimation of remaining tool life. The performances of fuzzy logic and ESNN with respect to diagnosis and prognosis of PCD tool wear has been compared.
\end{abstract}

Keywords: Fuzzy logic, Echostate neural network, Prognosis, Diagnosis, Tool wear

\section{INTRODUCTION}

Aluminium matrix composites (AMCs)[1-4] refer to the class of light weight high performance aluminium centric material systems. The reinforcement in AMCs could be in the form of continuous / discontinuous fibres, whisker or particulates, in volume fractions ranging from a few percent to $70 \%$. Properties of AMCs can be tailored to the demands of different industrial applications by suitable combinations of matrix, reinforcement and processing route. Presently several grades of AMCs are manufactured by different routes. Three decades of intensive research have provided a wealth of new scientific knowledge on the intrinsic and extrinsic effects of ceramic reinforcement vis-a-vis physical, mechanical, thermo-mechanical and tribological properties [5-10] of AMCs. In the last few years, AMCs have been utilized in high-tech structural and functional applications including aerospace, defence, automotive, and thermal management areas, as well as in sports and recreation. It is interesting to note that research on particle-reinforced cast AMCs took root in India during the 70 's, attained industrial maturity in the developed world and is currently in the process of joining the mainstream of materials.

This paper presents the method of using fuzzy logic with echostate neural network to estimate (diagnosis) the amount of tool wear during machining of AISiC with PCD. During the process, the amount of remaining life time has to be estimated. Diagnosis refers to estimation of amount of tool worn out and prognosis refers to estimation of remaining tool life.

Tool wear is the process repeated rubbing of sides of a tool, in this work it is PCD against the AISiC metal matrix composite workpiece. The tool wear has been categorized into different ranges (Table 1).

Table 1. Tool wear classification

\begin{tabular}{|l|l|}
\hline$\leq 0.1$ & Low wear \\
\hline 0.1 to 0.2 & Medium wear \\
\hline 0.2 to 0.25 & High wear \\
\hline Greater than 0.25 & Results into chatter \\
\hline
\end{tabular}

\section{MATERIALS AND METHODS}

\section{A. Properties of $A A 6061$}

The following are important properties of Al6061 metal matrix work piece (Table 2).

\section{B. Experimental Setup}

The tool used is a single point indexable tip. PCD contains a small amount of cobalt as a result of the manufacturing process. If a PCD tool is subjected continuous and significant heating during cutting, the diamond is likely to transform back to graphite. In order to avoid this effect, the use of coolant is recommended. Due to the polycrystalline nature of $P C D$, it is impossible to create cutting edges as perfect as those of single crystal diamond. Even with the finest grade 
Table 2. Properties of work piece

\begin{tabular}{|l|c|c|}
\hline \multicolumn{1}{|c|}{ Properties } & Values & Conditions $\mathrm{T}\left({ }^{\circ} \mathrm{C}\right)$ \\
\hline Density $\left(\times 1000 \mathrm{~kg} / \mathrm{m}^{3}\right)$ & 2.7 & 25 \\
\hline Poisson's Ratio & 0.33 & 25 \\
\hline Elastic Modulus (GPa) & $70-80$ & 25 \\
\hline Tensile Strength (Mpa) & 115 & 25 \\
\hline Yield Strength (Mpa) & 48 & 25 \\
\hline Elongation (\%) & 25 & 25 \\
\hline Hardness (HB500) & 30 & 25 \\
\hline Shear Strength (MPa) & 83 & 25 \\
\hline Fatigue Strength (MPa) & 62 & 25 \\
\hline $\begin{array}{l}\text { Thermal Expansion } \\
\left(10^{-6} /{ }^{\circ} \mathrm{C}\right)\end{array}$ & 23.4 & $20-100$ \\
\hline $\begin{array}{l}\text { Thermal Conductivity } \\
(\text { W/m-K) }\end{array}$ & 180 & 25 \\
\hline $\begin{array}{l}\text { Electric Resistivity } \\
\left(10^{-9} \text { W-m }\right.\end{array}$ & 37 & 25 \\
\hline
\end{tabular}

Properties of polycrystal diamond (Table 3). Accepted wear limit for PCD is $0.25 \mathrm{~mm}$

Table 3. Properties of PCD tool

\begin{tabular}{|l|c|c|}
\hline Density $(\mathrm{g} / \mathrm{cc})$ & 3.43 & 3.49 \\
\hline Compressive strength (GPa) & 4.74 & $4.15-5.33$ \\
\hline knoop hardness (GPa) & 50 & $44-60$ \\
\hline
\end{tabular}

PCD, which has a particle size of 2 microns, it is not possible to machine plastics and produce optically flat surfaces.

PCD tools are relatively expensive, compared with conventional cutting tools. Poor quality materials, which have inclusions that break conventional cutting tools, or work holding systems that do not locate and hold the part securely, are likely to have the same effect on PCD tools but at a greater cost.

Metal matrix composite (MMC) materials, Aluminium reinforced with Silicon carbide particles or filaments can be machined with PCD, but as the SiC content increases the tool life reduces and materials with more than $30 \%$
$\mathrm{SiC}$ are practically impossible to machine other than by grinding. The size of the work piece is $55 \times 250 \mathrm{~m}$ length. The turning experiments were conducted and the readings are given in Table 4 .

\section{Fuzzy Logic(FL)}

Fuzzy logic has rapidly become one of the most successful of today's technologies for developing sophisticated control systems. The reason for which is very simple. Fuzzy logic addresses such applications perfectly as it resembles human decision making with an ability to generate precise solutions from certain or approximate information. It fills an important gap in engineering design methods left vacant by purely mathematical approaches (e.g. linear control design), and purely logic-based approaches in system design. While other approaches require accurate equations to model real-world behaviors, fuzzy design can accommodate the ambiguities of real-world human language and logic. It provides both an intuitive method for describing systems in human terms and automates the conversion of those system specifications into effective models.

\section{Echostate Neural Network (ESNN)}

An artificial neural network (ANN) is an abstract simulation of a real nervous system that contains a collection of neuron units, communicating with each other via axon connections. Such a model bears a strong resemblance to axons and dendrites in a nervous system. Due to this self-organizing and adaptive nature, the model offers potentially a new parallel processing paradigm. This model could be more robust and user-friendly than the traditional approaches. ANN can be viewed as computing elements, simulating the structure and function of the biological neural network. These networks are expected to solve the problems, in a manner which is different from conventional mapping. Neural networks are used to mimic the operational details of the human brain in a computer. Neural networks are made of artificial 'neurons', which are actually simplified versions of the natural neurons that occur in the human brain. It is hoped, that it would be possible to replicate some of the desirable features of the human brain by constructing networks that consist of a large number of neurons. A neural architecture comprises massively parallel adaptive elements with interconnection networks, which are structured hierarchically. 
Dynamic computational models require the ability to store and access the time history of their inputs and outputs. The most common dynamic neural architecture is the time-delay neural network (TDNN) that couples delay lines with a nonlinear static architecture where all the parameters (weights) are adapted with the backpropagation algorithm. Recurrent neural networks (RNNs) implement a different type of embedding that is largely unexplored. RNNs are perhaps the most biologically plausible of the ANN models. One of the main practical problems with RNNs is the difficulty to adapt the system weights. Various algorithms, such as back propagation through time and real-time recurrent learning, have been proposed to train RNNs. These algorithms suffer from computational complexity, resulting in slow training, complex performance surfaces, the possibility of instability, and the decay of gradients through the topology and time. The problem of decaying gradients has been addressed with special processing elements (PEs).

The echostate network (ESNN)[11-13], Figure 1, with a concept new topology has been found by. ESNNs possess a highly interconnected and recurrent topology of nonlinear PEs that constitutes a "reservoir of rich dynamics" and contain information about the history of input and output patterns. The outputs of these internal PEs (echostates) are fed to a memory less but adaptive readout network (generally linear) that produces the network output. The interesting property of ESNN is that only the memory less readout is trained, whereas the recurrent topology has fixed connection weights. This reduces the complexity of RNN training to simple linear regression while preserving a recurrent topology, but obviously places important constraints in the overall architecture that have not yet been fully studied.

The echostate condition is defined in terms of the spectral radius (the largest among the absolute values of the eigenvalues of a matrix, denoted by $(\mathrm{I} \mid \mathrm{I})$ of the reservoir's weight matrix $(\|W\|<1)$. This condition states that the dynamics of the ESNN is uniquely controlled by the input, and the effect of the initial states vanishes. The current design of ESNN parameters relies on the selection of spectral radius. There are many possible weight matrices with the same spectral radius, and unfortunately they do not all perform at the same level of mean square error (MSE) for functional approximation.

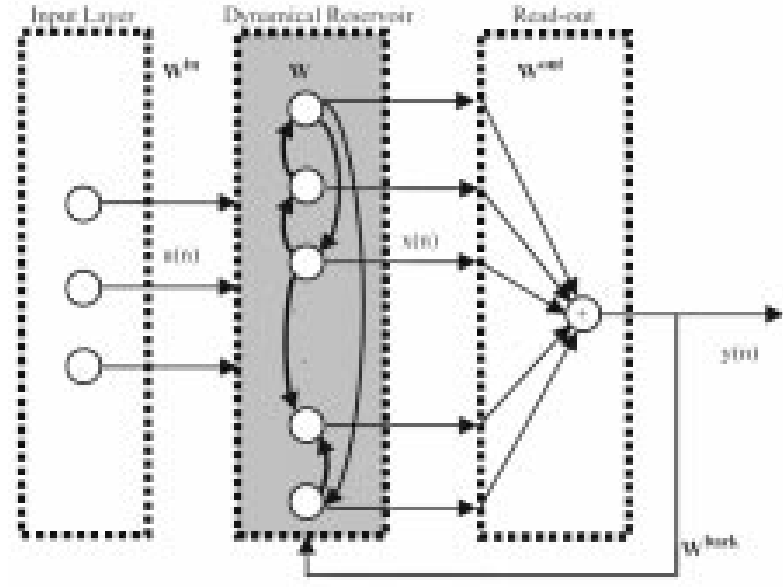

Fig. 1. An echostate network (ESNN)

To train the ESNN, reservoirs and state matrix have to be used. The number of the iterations required for ESNN is lesser than the number of iterations required for SDM.

The recurrent network is a reservoir of highly interconnected dynamical components, states of which are called echostates. The memory less linear readout is trained to produce the output. Consider the recurrent discrete-time neural network given in Figure 1 with $M$ input units, $\mathrm{N}$ internal PEs, and $\mathrm{L}$ output units.

The value of the input unit at time $n$ is

$$
u(n)=\left[u_{1}(n), u_{2}(n), \ldots, u_{M}(n)\right]^{T} \quad \ldots \text { (i) }
$$

The internal units are

$$
\begin{aligned}
x(n)= & {\left[x_{1}(n), x_{2}(n),, x_{M}(n)\right]^{T} \quad \ldots \text { (ii) } } \\
& , \text { and output units are } \\
y(n)= & {\left[y_{1}(n), y_{2}(n), \ldots, y_{L}(n)\right]^{T} \quad \ldots \text { (iii). } }
\end{aligned}
$$

The connection weights are given

- in an $(N \times M)$ weight matrix $W^{\text {in }}=W^{\text {in }}$ for connections between the input and the internal PEs,

- in an $N \times N$ matrix $W^{\text {out }}=W^{\text {in }}$ for connections between the internal PEs

- in an $L \times N$ matrix $W^{\text {out }}=W^{\text {out }}$ for connections from PEs to the output units and 
- in an $N \times L$ matrix $W^{\text {back }}=W_{\text {if }}^{\text {back }}$ for the connections that project back from the output to the internal PEs.

The activation of the internal PEs (echostate) is updated according to

$x(n+1)=f\left(W^{\text {in }} u(n+1)+W x(n)+W^{\text {pack }} y(n)\right)$

where $f=\left(f_{1}, f_{2}, \ldots, f_{N}\right)$ are the internal PEs' activation functions.

Here, all $f_{j}^{\prime}$ s are hyperbolic tangent functions.

$$
\frac{e^{x}-e^{-x}}{e^{x}+e^{-x}}
$$

The output from the readout network is computed according to

$$
y(n+1)=\rho^{\text {out }}\left(W^{\text {out }} x(n+1)\right),
$$

where

$\rho^{\text {out }}=\left(\rho_{1}^{\text {out }}, \rho_{2}^{\text {out }}, \ldots, \rho_{L}^{\text {ut }}\right)$ are the output unit's nonlinear functions [15-16] Generally, the readout is linear so foutis identity.

\section{IMPLEMENTATION}

\section{A. Fuzzy logic training and testing}

Generate a Sugeno-type Fuzzy logic information system using subtractive clustering using the inputs

1. Volume Percentage, 2. Speed - $\mathrm{m} / \mathrm{min}$, 3. Feed rate - $\mathrm{mm} / \mathrm{rev}, 4$. Depth of cut $\mathrm{mm}$, 5. Machining time min, 6. Flank wear land width $\left(v_{b}\right)$ $\mathrm{mm}, 7$. Specific energy $w / \mathrm{mm}^{3} / \mathrm{mm}$ and target output, Surface roughness, Ra, micrometer

\section{B. ESNN training and testing}

Training echostate network

Decide the input features of the registered image

Fix the target values

Set no. of inputs $=7$;

Set no of reservoir $=20$;

Set no. of output $=1$
Create weight matrix(no of reservoirs, number of inputs) $=$ random numbers -0.5

Create weight backup matrix (number of outputs, no of reservoirs) $=($ random numbers -0.5$) / 2$

Create weight not (w0) (number of reservoirs, no of reservoirs) $=$ (random numbers -0.5 )

Create temp matrix (te) (no.of reservoirs, no of reservoirs) $=$ random numbers

Calculate w0 w0. * (te $<0.3$ )

Calculate $w 0=w 0{ }^{*}(w 0<0.3)$

Follow the heuristics

$$
\begin{aligned}
& \mathrm{v}=\text { eig (w0) } \\
& \text { lambda = max (abs(v)) } \\
& \mathrm{w} 1=\mathrm{w} 0 / \text { lambda } \\
& \mathrm{w}=.9^{*} \mathrm{w} 1
\end{aligned}
$$

Create network training dynamics

state $=$ zeros $($ no_reservoir, 1$)$

desired $=0$;

for loop

input $=x(i: i+n i p p-1)$

$F=w_{t}$ input $^{\star}$ input'

$\mathrm{TT}=\mathrm{W}^{*}$ state

TH=wt_back' * desired

next_state $=\tanh (\mathrm{F}+\mathrm{TT}+\mathrm{TH})$

state $=$ next_state

desired $=x(i+n i p p-1)$

desired_1 = desired

end

Network testing

input $=x(i: i+n i p p-1)$;

$F=w_{t}$ input $^{\star}$ input';

TTH=wt_back' * output_d;

next_state $=\tanh \left(F+w^{*}\right.$ state $\left.+T T H\right)$;

state $=$ next_state;

output (i) = (wout'*state);

\section{RESULTS AND DISCUSSION}

Figure 2 presents the plot for the actual output and estimated outputs by fuzzy logic. The amount of deviation between the estimated output and the actual output is minimum. The $x$ axis represents tool machining data and the $y$ axis represents estimation by fuzzy logic. 
Kathirvel et al : Comparison of Performances of Fuzzy Logic...

Table 4 Experimental data

\begin{tabular}{|c|c|c|c|c|c|c|c|c|c|c|c|}
\hline S.No & Volume & fraction $\%$ & $\begin{array}{l}\text { Speed } \\
\mathrm{m} / \mathrm{min}\end{array}$ & $\begin{array}{c}\text { Feed } \\
\mathrm{mm} / \mathrm{rev}\end{array}$ & $\begin{array}{c}\text { Depth } \\
\text { of cut, } \\
\mathrm{mm}\end{array}$ & $F_{X N} N$ & $F_{y} N$ & $\overline{F_{Z} N}$ & $\begin{array}{c}\text { Machining } \\
\text { time, } \\
\text { min }\end{array}$ & $\begin{array}{c}\text { Flank } \\
\text { wear } \\
V_{b}, \mathrm{~mm}\end{array}$ & \begin{tabular}{|c|} 
Specific \\
energy \\
$x_{3} 10$ \\
w.s $/ \mathrm{mm}$
\end{tabular} \\
\hline 1 & 10 & 50 & 0.2 & 0.5 & 35 & 65 & 70 & 2 & 0.03 & 1519 & 1.71 \\
\hline 2 & 10 & 50 & 0.4 & 1.5 & 40 & 65 & 75 & 5 & 0.14 & 20.47 & 3.83 \\
\hline 3 & 10 & 50 & 0.6 & 2.5 & 40 & 75 & 80 & 8 & 0.31 & 25.11 & 5.48 \\
\hline 4 & 10 & 100 & 0.2 & 1.5 & 50 & 75 & 90 & 5 & 0.15 & 13.53 & 1.85 \\
\hline 5 & 10 & 100 & 0.4 & 2.5 & 45 & 85 & 105 & 8 & 0.35 & 17.05 & 3.12 \\
\hline 6 & 10 & 100 & 0.6 & 0.5 & 60 & 90 & 110 & 2 & 0.04 & 11.88 & 2.65 \\
\hline 7 & 10 & 150 & 0.2 & 2.5 & 40 & 80 & 65 & 8 & 0.36 & 13.03 & 1.74 \\
\hline 8 & 10 & 150 & 0.4 & 0.5 & 45 & 80 & 70 & 2 & 0.04 & 9.362 & 1.73 \\
\hline 9 & 10 & 150 & 0.6 & 1.5 & 45 & 90 & 85 & 5 & 0.18 & 12.23 & 2.81 \\
\hline 10 & 15 & 50 & 0.2 & 1.5 & 50 & 90 & 85 & 8 & 039 & 38.24 & 2.11 \\
\hline 11 & 15 & 50 & 0.4 & 2.5 & 40 & 105 & 105 & 2 & 0.07 & 16.88 & 2.75 \\
\hline 12 & 15 & 50 & 0.6 & 0.5 & 45 & 110 & 105 & 5 & .19 & 41.03 & 3.17 \\
\hline 13 & 15 & 100 & 0.2 & 2.5 & 60 & 80 & 125 & 8 & 0.49 & 29.90 & 2.78 \\
\hline 14 & 15 & 100 & 0.4 & 0.5 & 55 & 85 & 130 & 5 & 0.21 & 21.30 & 1.01 \\
\hline 15 & 15 & 100 & 0.6 & 1.5 & 60 & 80 & 125 & 8 & 0.49 & 29.90 & 2.78 \\
\hline 16 & 15 & 150 & 0.2 & 0.5 & 55 & 85 & 130 & 5 & 0.21 & 21.30 & 1.01 \\
\hline 17 & 15 & 150 & 0.4 & 2.5 & 60 & 150 & 210 & 8 & 0.51 & 23.48 & 1.82 \\
\hline 18 & 15 & 150 & 0.6 & 2.5 & 60 & 100 & 115 & 2 & 0.09 & 10.09 & 2.02 \\
\hline 19 & 15 & 50 & 0.2 & 2.5 & 60 & 90 & 125 & 5 & 0.35 & 42.83 & 1.48 \\
\hline 20 & 25 & 50 & 0.4 & 0.5 & 55 & 85 & 140 & 8 & 0.56 & 87.50 & 1.91 \\
\hline 21 & 25 & 50 & 0.6 & 1.5 & 60 & 100 & 120 & 2 & 0.11 & 32.88 & 2.27 \\
\hline 22 & 25 & 100 & 0.2 & 0.5 & 25 & 70 & 85 & 8 & 0.62 & 57.86 & 0.93 \\
\hline 23 & 25 & 100 & 0.4 & 1.5 & 60 & 100 & 125 & 2 & 0.12 & 22.34 & 1.30 \\
\hline 24 & 25 & 100 & 0.6 & 2.5 & 70 & 100 & 115 & 5 & 0.43 & 33.49 & 1.95 \\
\hline 25 & 25 & 150 & 0.2 & 1.5 & 50 & 80 & 105 & 2 & 0.12 & 17.07 & 0.72 \\
\hline 26 & 25 & 150 & 0.4 & 2.5 & 50 & 85 & 100 & 5 & 0.45 & 26.29 & 1.28 \\
\hline 27 & 25 & 150 & 0.6 & 0.5 & 25 & 70 & 90 & 8 & 0.72 & 52.28 & 1.40 \\
\hline
\end{tabular}




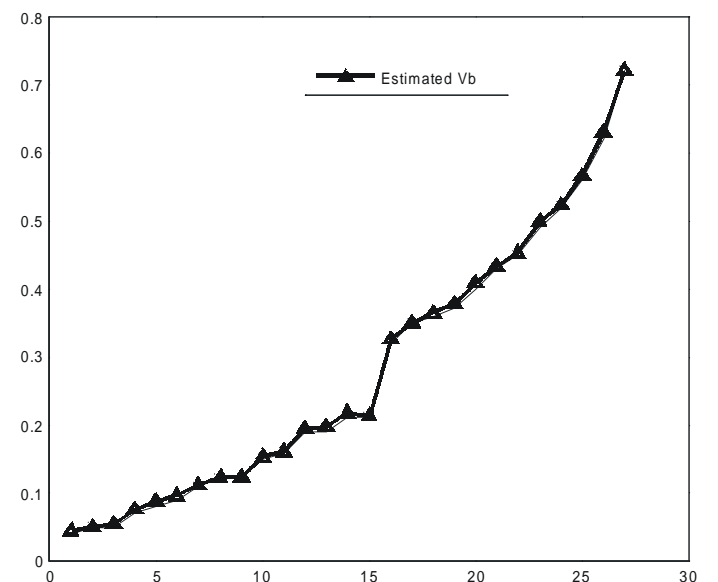

Fig. 2 Prediction of flank wear by using Fuzzy logic

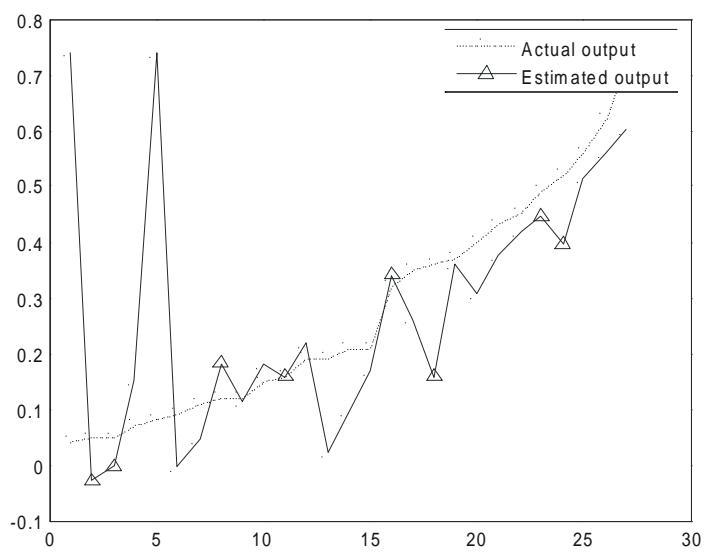

Fig. 3 Estimation of $V_{b}$ using ESNN considering cutting forces

Table 5. Comparison of performances of Fuzzy logic with Echostate neural network in diagnosis and prognosis of tool wear of PCD

\begin{tabular}{|l|l|c|c|c|}
\hline \multicolumn{1}{|c|}{ Algorithm } & \multicolumn{1}{|c|}{ Empirical formula } & $\begin{array}{c}\text { Computational } \\
\text { effort }\end{array}$ & $\begin{array}{c}\text { Percentage of } \\
\text { Diagnosis }\end{array}$ & $\begin{array}{c}\text { Percentage of } \\
\text { Prognosis }\end{array}$ \\
\hline Fuzzy logic & $n_{p}^{2 *} n_{f} / n_{c} n_{f}+\left(n_{f}-1\right)$ & 16585 & 100 & 100 \\
\hline Echostate & $n_{p} n_{h} n_{i}\left(n_{h} n_{o}+n_{i}+\right.$ & 116640 & 90.62 & 90.62 \\
neural network & $n_{o}\left(n_{i}+n_{h}\right)$ & & & \\
\hline
\end{tabular}

Figure 3 presents the plot for the actual output and estimated outputs by ESNN. The amount of deviation between the estimated output and the actual output is marginal. The $x$-axis represents tool machining data and the $y$-axis represents estimation by ESNN.

Table 5 presents the comparisons of performances of fuzzy logic with echostate neural network in diagnosis and prognosis of tool wear of PCD while machining of $\mathrm{Al}$ metal matrix composites. In the table, it can noticed that, the computational complexity (third column) of fuzzy logic is less than that of ESNN and percentage of diagnosis and prognosis is 100\% (fourth and fifth column) in case of fuzzy logic than that of ENN which is only $85.19 \%$. The fuzzy logic appears to be better than that of echostate neural network for the machining data presented in Table 4. The situation may change for a different sets of data.

\section{CONCLUSIONS}

In this research work, diagnosis and prognosis of tool wear of PCD on Al MMC machining and their machinability behavior were analyzed using fuzzy logic and echostate neural network. The percentage of diagnosis and prognosis is better through fuzzy logic than that of ESNN.

\section{REFERENCES}

[1] J.Paulo Davim, N.Muthukrishnan, Optimization of machining parameters of Al/SiC-MMC with ANOVA and ANN analysis, J. Materials Processing Technology (2008)

[2] N.Muthukrishnan, M.Murugan, K.Prahlada Rao, An Investigation on the machinability of AISiC metal matrix composites using PCD inserts, Int. J Adv Manuf Technology 2007

[3] K.Palanikumar, R.Karthikeyan, Assessment of factors influencing surface roughness on the machining of AISiC particulate composites (2006)

[4] A. Ibrahim, F.A.Mohamed, E.J.Lavernia, Particulate reinforced metal matrix composites-a review, J. Mat. Sci. 26 (1991) 1137-1156. 
[5] F.Dolauy, Increasing focus on silicon carbide-reinforced aluminum composites, Light Metal Age 6 (1986) 7-14.

[6] N.Tmoac, K.Tonnessen, Machining of particulate aluminium matrix composites, Ann. CIRP 41 (1) (1992) 55-58.

[7] K.Weinert, A consideration of tool wear mechanism when machining metal matrix composites, Ann. CIRP 42 (1) (1993) 95-98.

[8] G.A.Chadwick, P.J.Health, Machining metal matrix composites, Met. Mat. 2 (1990) 73-76.

[9] M.K.Brun, M.Lee, Wear characteristics of various hard materials for machining SiC-reinforced aluminum alloy, Wear 104 (1985) 21-29.
[10] L.Cronjage, D.Merister, Machining of fibre and particle-reinforced aluminium, Ann. CIRP 41 (1) (1992) 63-66.

[11] H.Jaeger; The echo state approach to analyzing and training recurrent neural networks; (Tech. Rep. No. 148). Bremen: German National Research Center for Information Technology, 2001.

[12] H.Jaeger; Tutorial on training recurrent neural networks, covering BPPT, RTRL,EKF and the "echo state network" approach (Tech. Rep. No. 159).; Bremen: German National Research Center for Information Technology, 2002.

[13] H.Jaeger;. Short term memory in echo state networks; (Tech. Rep. No. 152) Bremen: German National Research Center for Information Technology. 2002. 